\title{
The Relevance of the English Antonyms
}

\author{
Rui Li \\ School of Foreign Languages \\ Henan University of Chinese Medicine \\ Zhengzhou, Henan, China
}

\begin{abstract}
Antonym has been a hot issue in semantic study. There are three types of English antonyms: contrary antonym, complementary antonym and converse antonym. According to the traditional linguistics, antonyms are totally opposite in meaning. This paper will challenge this traditional view and explore the relevance of antonyms following the three types of antonyms, hoping to reveal the nature of antonyms in some degree. Then on the basis of that, finally this paper will draw some criterions of English antonyms.
\end{abstract}

Keywords-contrary antonym; complementary antonym; converse antonym; semantic field theory; hyponymy theory; componential analysis theory

\section{INTRODUCTION}

Antonym has been a hot issue in semantic study. Traditional semantics consider antonym simply means the oppositeness of meaning. But with the development of modern semantics, more scientific and detailed researches about antonym appeared. These researches, especially researches made by John Lyons, have shed new light on antonym studies. Now modern semantics classifies the antonyms into three types on the basis of semantic opposition: contrary antonym, complementary antonym and converse antonym. In order to challenge the traditional view that antonyms are totally opposite in meaning, this paper tries to discuss the relevance of antonyms following the three types of antonyms, hoping to reveal the nature of antonyms in some degree. Then on the basis of the relevance of antonyms, finally this paper will draw some criterions of English antonyms.

\section{CONTRARY ANTONYM}

Contrary antonym shows a type of oppositeness of meaning, illustrated by such pairs as wide/narrow, old/young, big/small, etc. They can be seen in terms of degrees of the quality involved. Thus a road may be wide or very wide and may be wider than another. This shows that the semantic polarity in contrary antonyms is relative and the opposition is graded. Because of this, contrary antonyms are also known as gradable antonyms in some linguistic books. This means that contrary antonyms can be placed at both extremes of a scale, between which there may be gradable lexical items. For example, between the antonymic pair beautiful - ugly, there may be such gradable adjectives as:

$$
\text { beautiful-pretty - good-looking - plain-ugly. }
$$

Between the antonymic pair love - hate, there may be such gradable nouns as:

$$
\text { love - attachment - liking - indifference - antipathy }
$$
- hate.

Beside the oppositeness of meaning, do these contrary antonyms have something in common? Now we turn to some semantic theories for the explanation of this question.

In semantics, we use the term "Semantic Field" to refer to a type of paradigmatic relations. In any language, words sharing the same concept can be classified into meaning areas or semantically related sets. The semantic field is the organization of words as a system of interrelated lexical networks, and words of a semantic field are joined together by a common concept. For example, the color terms in English: green, blue, black, gray, orange, rose, olive, purple, lemon, emerald, sandy, coral, and so on. A list of words referring to items of a particular class like this is called a semantic field. So according to semantic field theory, contrary antonyms must be words that belong to one semantic field, stay in opposite directions and have the same distance to the average level. To illustrate this relationship clearly, look at the following minor semantic field.

$$
\text { hot - warm - tepid - lukewarm - cool-cold }
$$

The above is a minor semantic field about temperature. Between the extreme antonym---hot and cold, there are warm and cool, which also form a pair of antonyms themselves, and may have a further intermediate pair of antonym---tepid and lukewarm. All the three groups of antonyms have the same distance to the average level in this minor semantic field.

\section{COMPLEMENTARY ANTONYM}

Complementary antonyms represent a type of binary semantic contrast, that is, the members of a pair in this type are complementary to each other, e.g. male - female, boy - girl, etc. Male is said to be the complementary of female, and vice versa. In such a relationship, the assertion of one of the items implies the denial of the other and the denial of one of the items implies the assertion of the other.

Then what is the relevance of the English antonyms? Just like the contrary antonym, we can also use semantic field theory to define and describe this kind of antonym. These complementary antonyms must be in the same semantic field. 
But different from the contrary antonyms, they divide up the whole of a semantic field completely in two parts. For example, male - female they belong to the same semantic field: sex terms of human, and they divide up the whole of this semantic field completely.

Then we can also turn to hyponymy theory for the answer of the above question. Concerning sense relation, there is a special relation termed as hyponymy in semantics, meaning inclusiveness. To be more specific, it refers to a relationship of a group of words, in which the meaning of one of the words includes the meaning of the other words. For example, in English the words animal and $\operatorname{dog}$ are related in such a way that $d o g$ refers to a kind of animal, and animal is a general term. That is to say, if any object is a $d o g$, then it is necessarily an animal, so the meaning of animal is included in the meaning of $\operatorname{dog}$ (note, the class membership is opposite to meaning inclusion: the class of animals includes the class of dogs). The specific term, dog, is called a hyponym, and the general term, animal, is called a superordinate. A superordinate term can have many hyponyms. The members of the same superordinate are called co-hyponyms. So according to hyponymy theory, complementary antonyms must be co-hyponyms under the same superordinate. For the pair of antonym of "man and woman", "man" and "woman" are co-hyponyms under the same superordinate "human".

What's more, if we use componential analysis theory in semantics, we can also find the similarity of these antonyms. Componential analysis is an approach to the study of meaning which analyses a word into a set of meaning components or semantic features. Semantic features constitute the linguistic meaning of a word. They are a set of abstracted characteristics that distinguish the category that the word names from all other categories. Semantic features are established on the basis of binary opposition. For example, we can factor out Male/Female as the binary opposition that holds between the noun pairs man and woman, boy and girl.

$$
\begin{aligned}
& \text { man [+Human, +Adult, +Male] } \\
& \text { woman [+Human, +Adult,-Male] } \\
& \text { boy [+Human, -Adult, +Male] } \\
& \text { girl [+ Human,-Adult, -Male] }
\end{aligned}
$$

Through the above componential analysis, we can see that the antonym "man and woman" have the same semantic features [+Human], [+Adult]. The only oppositeness is man is [+Male], while woman is [-Male]. This is also the case of the antonyms "boy and girl". So we can draw a conclusion that the complementary antonyms must only have one opposite semantic feature. In this sense, we can also say "man and boy" is a pair of antonym, "woman and girl" is a pair of antonym, since both of them only have oppositeness in $[ \pm$ Adult $]$.

\section{CONVERSE ANTONYM}

This is a special type of antonym in that the members of a pair do not constitute a positive-negative opposition. They show the reversal of a relationship between two entities. This kind of relationship can be illustrated by such as buy - sell, give - receive, parent - child, debtor - creditor, above below, etc. Buy is said to be the converse of sell and vice versa: If $A$ sells a watch to $B, B$ buys a watch from $A$. The same applies to the pair above - below: If $\mathrm{A}$ is above $\mathrm{B}, \mathrm{B}$ is below A. This type of antonym is typically seen in reciprocal social roles, spatial relationship and so on.

If we examine carefully, this type of antonyms are not opposite absolutely, there is an interdependence of meaning, such that one member of the pair presupposes the other member. If there is no "buy", there will be no "sell". If there is no above, there will be no "below". If there is no "parent", there will be no "child". . In this respect, "converseness" contrasts with "complementarity", where there is no such symmetry of dependence. This is the major difference between this type and the previous two.

\section{CONCLUSION}

Through the above semantic analysis, we can draw a conclusion that antonyms don't simply means the oppositeness of meaning, but they are related to each other to some extent. That is, they have many aspects of relevance but only one point of oppositeness.

To be more specific, for the three types of English antonyms, contrary antonyms must belong to one semantic field in which there is a gradable relationship between them. Complementary antonyms divide up the same semantic field completely in two parts, and they are complementary to each other; they are co-hyponyms under the same superordinate; they have many same semantic features, and only one opposite semantic feature. Converse antonyms have an interdependence of meaning, such that one member of the pair presupposes the other member.

After the analysis of the relevance of the English antonyms, we can further draw some basic criterions of the English antonyms:

- Antonyms must belong to the same semantic field. That is, antonyms must be words that are opposite in meaning while having relevance from logic perspective so that they can be compared. For example, person and idea can't be antonyms, because they don't belong to one same semantic field, and they are incomparable. Only some words such as light-heavy, teacher-student can be antonyms, because they are related in logic and opposite in meaning.

- If we use componential analysis theory, the antonyms must have many same semantic features, and only one opposite semantic feature

- Antonyms should be two contrary, converse or contradictary co-hyponyms under the same superordinate. 


\section{REFERENCES}

[1] John I. Saeed. Semantics. [M]. Beijing: Foreign Language Teaching and Research Press, 2000.

[2] Lyons J. An Introduction to Theoretical Linguistics. [M].Cambridge: Cambridge University Press, 1968.

[3] Stuart C. Poole. An Introduction to Linguistics. [M].Beijing: Foreign Language Teaching and Research Press,2000.

[4] Hu Zhuanglin. Linguistics [M]. Beijing: Peking University Press, 2001

[5] Li Fuyin. Semantics. [M]. Beijing: Peking University Press, 1993.

[6] Wang Songhuai. On Semantica Feature of the English Antonyms. [J]. Journal of Higher Correspondence, 2007 (12). 